\title{
OPEN Embryonic periventricular endothelial cells demonstrate a unique pro-neurodevelopment and anti-inflammatory gene signature
}

Franciele Cristina Kipper ${ }^{1,5,7}$, Cleide Angolano ${ }^{2,5,7}$, Ravi Vissapragada ${ }^{1,4}$, Mauricio A. Contreras ${ }^{3}$, Justin Moore ${ }^{1,7}$, Manoj Bhasin ${ }^{6}$, Christiane Ferran ${ }^{2,3,5,7,8}$ \& Ajith J. Thomas ${ }^{1,5,7,8 \bowtie ~}$

Brain embryonic periventricular endothelial cells (PVEC) crosstalk with neural progenitor cells (NPC) promoting mutual proliferation, formation of tubular-like structures in the former and maintenance of stemness in the latter. To better characterize this interaction, we conducted a comparative transcriptome analysis of mouse PVEC vs. adult brain endothelial cells (ABEC) in mono-culture or NPC co-culture. We identified $>6000$ differentially expressed genes (DEG), regardless of culture condition. PVEC exhibited a 30-fold greater response to NPC than ABEC (411 vs. 13 DEG). Gene Ontology (GO) analysis of DEG that were higher or lower in PVEC vs. ABEC identified "Nervous system development" and "Response to Stress" as the top significantly different biological process, respectively. Enrichment in canonical pathways included HIF1A, FGF/stemness, WNT signaling, interferon signaling and complement. Solute carriers (SLC) and ABC transporters represented an important subset of DEG, underscoring PVEC's implication in blood-brain barrier formation and maintenance of nutrient-rich/ non-toxic environment. Our work characterizes the gene signature of PVEC and their important partnership with NPC, underpinning their unique role in maintaining a healthy neurovascular niche, and in supporting brain development. This information may pave the way for additional studies to explore their therapeutic potential in neuro-degenerative diseases, such as Alzheimer's and Parkinson's disease.

The development of the central nervous system (CNS) involves close orchestration between neural and vascular components ${ }^{1}$. During embryogenesis, neural tube development $(\sim$ E7.5-E9.5 in mouse $)$ precedes and promotes the formation of circumscribing perineural or pial vascular plexuses ${ }^{2}$, which give rise to brain vessels. Starting at E9.5, vascularization of the mouse neural tube results in the formation of periventricular vessels, originating from the telencephalic floor of the basal ganglia primordium, which in turn give rise to the embryonic forebrain arterial network by E11.5. Neurogenesis and angiogenesis in the embryonic brain are tightly coupled processes ${ }^{2-4}$.

Studies elucidating the role and function of the brain neurovascular unit (NVU) in health and disease have resulted in a paradigm shift from an exclusively "neurocentric" perspective to a more integrative process that emphasizes the dynamic cross-talks within the NVU between endothelial cells (EC), neurons, astrocytes, pericytes, and microglia ${ }^{4-7}$. EC-regulated self-renewal and proliferation of neural progenitor cells (NPC) was observed in cerebral vascular niches ${ }^{7,8}$. The NVU also regulates local blood flow and integrity of the blood-brain

${ }^{1}$ Division of Neurosurgery, Beth Israel Deaconess Medical Center, Boston, MA 02215, USA. ${ }^{2}$ Division of Vascular and Endovascular Surgery, Department of Surgery, Beth Israel Deaconess Medical Center, Boston, MA 02215, USA. ${ }^{3}$ Division of Vascular Surgery, Department of Surgery, Beth Israel Deaconess Medical Center, Boston, MA 02215, USA. " Discipline of Surgery, College of Medicine and Public Health, Flinders University, Adelaide 5042, SA, Australia. ${ }^{5}$ Center for Vascular Biology Research, Beth Israel Deaconess Medical Center, Boston, MA 02215, USA. ${ }^{6}$ BIDMC Genomics, Proteomics, Bioinformatics and Systems Biology Center, Beth Israel Deaconess Medical Center, Boston, MA 02215, USA. ${ }^{7}$ Harvard Medical School, Boston, MA 02215, USA. ${ }^{8}$ These authors jointly supervised this work: Christiane Ferran and Ajith J. Thomas. ${ }^{\circledR}$ email: athomas6@bidmc.harvard.edu 
barrier (BBB) via intricate signaling between its different components ${ }^{9-11}$. Prior studies exploring the symbiotic modulation of neurogenesis and angiogenesis in the context of neurodegeneration were primarily based on adult brain endothelial cells (ABEC), and hence failed to explore the potentially superior, and certainly more physiologic ability of embryonic brain EC to influence neuroangiogenesis ${ }^{12,13}$. We previously showed that embryonic brain periventricular EC (PVEC) surpass ABEC ability to promote NPC proliferation while delaying its differentiation ${ }^{4}$, although the identity and role of PVEC remained poorly understood. To address this gap, we performed a comparative RNA-seq based global gene expression analysis of PVEC vs. ABEC at baseline, and after co-culture with NPC. Our results show that ABEC and PVEC have a dramatically different transcriptome regardless of culture condition. At baseline, "transcription targets of Hypoxia Inducible Factor (HIF)-1" was the most modified pathway, with "WNT signaling" and "Interferon $\alpha / \beta$ signaling via JAK/STAT" being the most positively and negatively impacted pathways, respectively. Notably, solute carriers (SLC) and ABC transporters represented an important subset of differentially expressed genes (DEG), underscoring PVEC's potential role in BBB formation. Our results also strongly demonstrate that PVEC were significantly more prone to transcriptional changes than ABEC when co-cultured with NPC, further highlighting their prominent role in shaping the NVU during early embryonic brain development. Specifically, complement and immune response pathways were the most downregulated in PVEC following NPC co-culture, supporting the anti-inflammatory phenotype of these cells.

\section{Results}

Baseline gene expression profile shows major differences between PVEC and ABEC. PVEC were isolated from E15.5 mouse embryos, cultured until confluence, then sorted by CD31-coated magnetic beads, and characterized, as described ${ }^{4}$. PVEC and ABEC were cultured for $48 \mathrm{~h}$ prior to RNA extraction and subsequent RNAseq analysis. Each sample interrogation yielded 25-30 million reads. A particular gene was considered differentially expressed (DEG) if the $90 \%$ lower confidence bound of the fold change (FC) between samples was $>2$. Hierarchical clustering, using Pearson correlation distance metric on unsupervised RNAseq data showed perfect segregation based on EC bed (Fig. 1a).

The top 25 genes that were significantly higher in PVEC vs. ABEC included podoplanin $(P d p n)$, solute carrier (Slc) family 1 member 3 (Slc1a3), a-2-macroglobulin ( $A 2 m)$, membrane glycoprotein 6B (Gpm6b), insulin-like growth factor binding protein 5 (Igfbp5), neuropilin and tolloid like 2 (Neto2), myosin light chain 9 (Myl9) and glypican 4 (Gpc4) (Fig. 1b). Based on the literature, most of these genes reflect PVEC stemness and their ability to promote neural development ${ }^{14-16}$. The top 25 genes that were significantly lower in PVEC vs. ABEC included a number of EC-specific or EC-enriched molecules such as tyrosine kinase with immunoglobulin-like and EGF-like domains 1 (Tie1), platelet/endothelial cell adhesion molecule 1 (Pecam1), nitric oxide synthase 3 (Nos3), claudin 5 (Cldn5), lymphatic vessel endothelial hyaluronan receptor 1 (Lyve1), adhesion G-protein coupled receptor F5 (Adgrf5/Gpr116) and endomucin (Emcn) (Fig. 1b). These markers are highly expressed in mature adult brain $\mathrm{EC}^{17}$, hence their lower expression in PVEC implies the relative immaturity of these cells. Although ABEC and PVEC share a common endothelial identity and brain origin, a supervised analysis identified a staggering 5842 (2673 higher and 3169 lower) DEG between them, as depicted in the Volcano plot (Fig. 1c).

The PVEC transcriptome is enriched in genes that positively promote nervous system development and dampen inflammatory responses. We performed Gene Ontology biological process (GO_bp) analysis of DEG using Metacore from Clarivate Analytics. When analyzing transcripts that were higher in PVEC vs. ABEC, as expected, processes such as nervous system development, neurogenesis, generation of neurons, developmental processes and neuron differentiation were recognized among the top 5 most significant GO_bp. Analysis of transcripts that were lower in PVEC vs. ABEC identified processes such as response to stress, regulation of cytokine production and wound healing among the top 5 most significant GO_bp (Fig. 2a). In the combined datasets, the top 10 GO_bp were mostly related to those identified when using the "only higher" dataset, more specifically system development and nervous system development (Fig. 2b).

Canonical pathway enrichment also using the Metacore software identified pathways enriched in PVEC vs. ABEC. The top 3 most enriched pathways include "Transcription targets of Hypoxia Inducible Factor 1" (HIF-1), with hypoxia inducible factor 1, alpha subunit (Hifla) transcript levels being twofold lower in PVEC vs. ABEC, "Stem cells Schema: FGF signaling in embryonic stem cell renewal and differentiation", and "High shear stress platelet activation" (Fig. 2c). More than half of the genes in the HIF-1 pathway (54 of 95) were differentially expressed in PVEC vs. ABEC. Of these, 33/95 were $>$ twofold higher, and 21/95 were $>$ twofold lower (Table 1 and Supplementary Figure 2a). Specifically, transcript levels of: (a) angiogenesis-related vascular endothelial growth factor A (Vegfa, tenfold), fibroblast growth factor 2 (Fgf2, 2.3-fold), plasminogen activator inhibitor (PAI1/Serpine1, fourfold), thrombospondin 1 (Thbs1, 4.4-fold), and angiopoietin 2 (Angpt2, 4.8-fold) were higher, while those of chemokine (C-X-C motif) ligand 12 (Cxcl12/Sdf1, 14-fold), FMS-like tyrosine kinase 1 (Flt1/Vegfr1, 11-fold), endoglin (Eng, 13.7-fold), mast cell growth factor ( $M g f / K i t l, 8.4$-fold) and placental growth factor (Pgf/ Plgf, 8.5-fold) were lower; (b) regulators of extracellular matrix (ECM) remodeling matrix metallopeptidase 2 (Mmp2, 83.5-fold), lysyl oxidase-like 2 (Loxl2, 20.8-fold), and lysyl oxidase (Lox, 639-fold) were higher, while those of plasminogen activator urokinase receptor (Plaur, 3.4-fold), Loxl4 (18-fold) were lower; (c) regulators of glucose metabolism Slc2a3/Glut3 (421-fold), hexokinase 2 (Hk2, 16.5-fold) and Slc16a3/Mct4 (twofold) were higher, while hexokinase 1 ( $H k 1,2.3$-fold), enolase 1 (Eno1, 904-fold), phosphofructokinase, liver, B-type ( $P f k l, 2.4$-fold), and phosphoglycerate kinase 1 ( $P g k 1,2.4$-fold) were lower; (d) maintenance of stemness sex determining region Y (SRY)-box 2 (Sox2, 708-fold) was higher, while nanog homeobox (Nanog, ninefold) and POU domain, class 5, transcription factor 1 (Pou5f1/Oct-3/4, ninefold) were lower; (e) chemotaxis-associated chemokine (C-X3-C motif) receptor 1 (Cx3cr1, threefold) and Cxcr4 (401-fold) were higher, and (f) fibrosis/ 


\section{a}

b
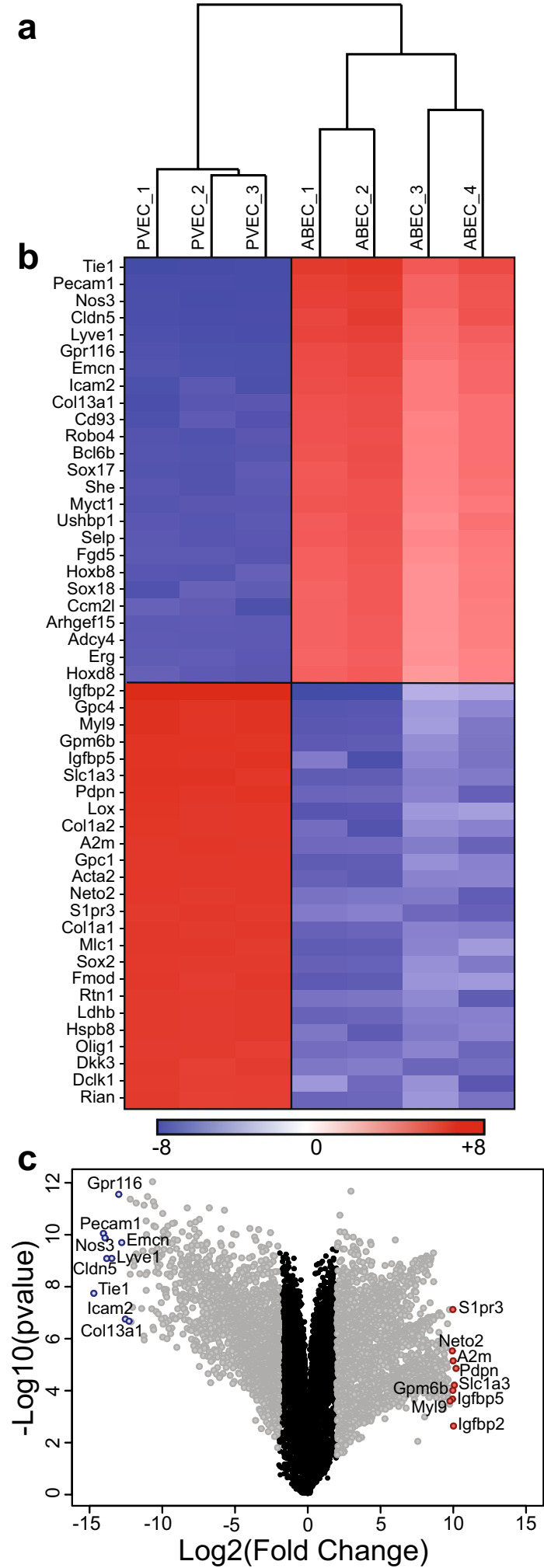

Figure 1. Baseline expression profile of PVEC and ABEC highlights the distinct identity of these vascular beds. (a) Hierarchical clustering indicates total segregation between PVEC and ABEC. (b) Heatmap of the top 50 genes that were significantly different in PVEC vs. ABEC, based on fold change. Red indicates genes with higher expression and blue indicates genes with lower expression in PVEC vs. ABEC. (c) Volcano plot encompassing all analyzed genes, depicted as dots. Black dots represent genes that were not significantly different, while gray dots represent genes that were significantly different in PVEC vs. ABEC, based on fold change (FC) $>2$ and $\mathrm{p}$-value $<0.05$. Highlighted in blue dots are the genes that were most significantly lower, and in red dots genes that were most significantly higher in PVEC vs. ABEC. 


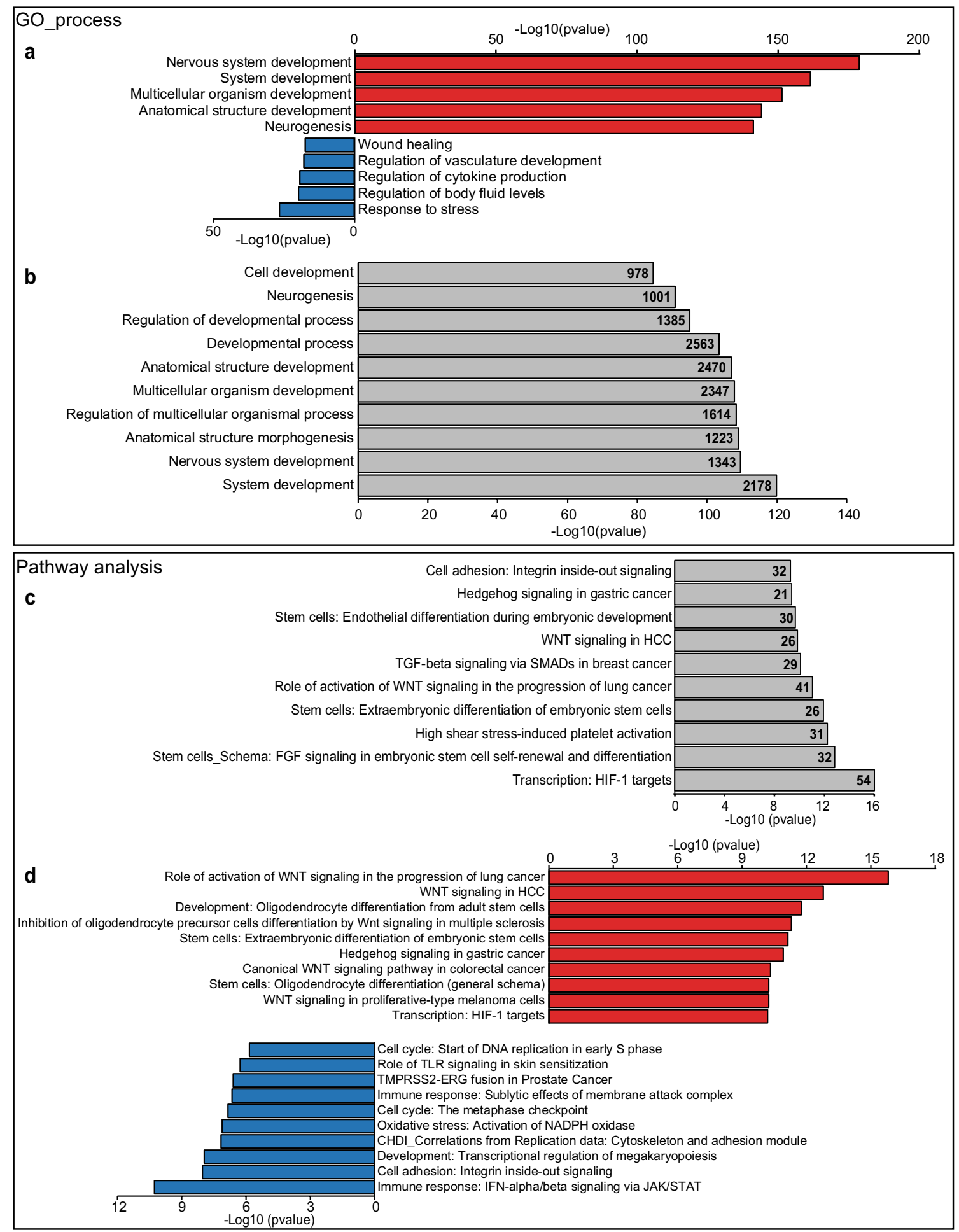

Figure 2. GO analysis identifies nervous system development and response to stress as the processes with the greatest number of DEG that were higher or lower in PVEC vs. ABEC. (a) Gene Ontology (GO) analysis using the Metacore from Clarivate Analytics identified the top 5 most enriched biological process when separated by higher (red) or lower (blue) differentially expressed genes (DEG) in PVEC vs. ABEC. (b) The top 10 more enriched biological processes when all DEG were analyzed together closely resembles the analysis performed using DEG that were higher in PVEC vs. ABEC. Numbers inside the bars correspond to the number of DEG in each given GO biological process. (c) Canonical pathway enrichment analysis using the Metacore identified the top 10 most enriched pathways in PVEC vs. ABEC, here depicted as grey bars. Numbers inside the bars correspond to the number of DEG that were identified in each given pathway. (d) Canonical pathway enrichment using the Metacore, following partitioning of our data into genes that were higher (red) or lower (blue) in PVEC vs. ABEC identified the top 10 pathways in each category. 


\begin{tabular}{|c|c|c|c|}
\hline Process & Gene & Fold change & p-value \\
\hline \multirow{12}{*}{ Angiogenesis } & VEGF-A & 10.14 & $1.15 \mathrm{E}-11$ \\
\hline & $\alpha 1 B$ adrenergic receptor & 7.34 & $5.64 \mathrm{E}-05$ \\
\hline & Angiopoietin 2 & 4.79 & $3.73 \mathrm{E}-09$ \\
\hline & Thrombospondin 1 & 4.40 & $6.40 \mathrm{E}-08$ \\
\hline & PAI & 4.09 & $9.08 \mathrm{E}-08$ \\
\hline & Adrenomedullin & 2.71 & $1.01 \mathrm{E}-07$ \\
\hline & FGF2 & 2.32 & $4.14 \mathrm{E}-02$ \\
\hline & MGF & -8.36 & $1.70 \mathrm{E}-10$ \\
\hline & PLGF & -8.52 & $1.77 \mathrm{E}-09$ \\
\hline & VEGFR-1 & -11.05 & $2.45 \mathrm{E}-09$ \\
\hline & Endoglin & -13.70 & $2.62 \mathrm{E}-11$ \\
\hline & SDF-1 & -14.11 & $1.21 \mathrm{E}-11$ \\
\hline \multirow{10}{*}{ Glucose metabolism } & GLUT3 & 421.30 & $1.27 \mathrm{E}-06$ \\
\hline & ALDOC & 74.49 & $6.35 \mathrm{E}-09$ \\
\hline & HXK2 & 16.55 & $4.62 \mathrm{E}-09$ \\
\hline & F263 & 2.87 & $7.85 \mathrm{E}-08$ \\
\hline & MCT4 & 2.00 & $1.35 \mathrm{E}-04$ \\
\hline & HXK1 & -2.32 & $9.61 \mathrm{E}-09$ \\
\hline & PGK1 & -2.43 & $2.08 \mathrm{E}-07$ \\
\hline & PFKL & -2.43 & $3.17 \mathrm{E}-09$ \\
\hline & PDK1 & -2.45 & $2.25 \mathrm{E}-07$ \\
\hline & ENO1 & -903.94 & $2.56 \mathrm{E}-13$ \\
\hline \multirow{6}{*}{ Extracellular matrix remodeling } & Lysyl oxidase & 638.91 & $7.68 \mathrm{E}-05$ \\
\hline & MMP-2 & 83.05 & $9.41 \mathrm{E}-10$ \\
\hline & LOXL2 & 20.82 & $4.43 \mathrm{E}-11$ \\
\hline & P4HA2 & -2.11 & $3.84 \mathrm{E}-08$ \\
\hline & \begin{tabular}{|l|} 
PLAUR \\
\end{tabular} & -3.36 & $1.31 \mathrm{E}-08$ \\
\hline & LOXL4 & -18.11 & $6.34 \mathrm{E}-09$ \\
\hline \multirow{4}{*}{ Fibrosis immuno-regulation } & TGF-beta3 & 33.51 & $7.20 \mathrm{E}-11$ \\
\hline & CTGF & 15.95 & $1.36 \mathrm{E}-09$ \\
\hline & TGF-beta2 & 9.03 & $1.27 \mathrm{E}-08$ \\
\hline & TGF-betal & -3.38 & $1.34 \mathrm{E}-08$ \\
\hline \multirow{3}{*}{ Stem cell maintenance } & SOX2 & 708.34 & $6.72 \mathrm{E}-06$ \\
\hline & Oct-3/4 & -9.17 & $6.98 \mathrm{E}-04$ \\
\hline & NANOG & -9.57 & $8.78 \mathrm{E}-08$ \\
\hline \multirow{2}{*}{ Cell cycle arrest } & $\mathrm{p} 21$ & 2.10 & $1.15 \mathrm{E}-08$ \\
\hline & c-Myc & -2.01 & $3.59 \mathrm{E}-07$ \\
\hline \multirow{2}{*}{ Chemotaxis } & CXCR4 & 400.55 & $3.39 \mathrm{E}-09$ \\
\hline & CX3CR1 & 3.17 & $1.35 \mathrm{E}-02$ \\
\hline \multirow{2}{*}{ Transcription regulation } & ROR-alpha & 100.74 & $1.49 \mathrm{E}-08$ \\
\hline & ID2 & 2.31 & $1.84 \mathrm{E}-08$ \\
\hline \multirow{2}{*}{ Nucleotide metabolism } & AK3 & 3.84 & $1.09 \mathrm{E}-07$ \\
\hline & $\mathrm{Nt5e}$ & -2.03 & $7.39 \mathrm{E}-05$ \\
\hline \multirow{2}{*}{ Iron ion metabolism } & Ceruloplasmin & 80.37 & $9.94 \mathrm{E}-12$ \\
\hline & Transferrin & 61.58 & $4.14 \mathrm{E}-08$ \\
\hline Regulation of $\mathrm{pH}$ & Carbonic anhydrase IX & 11.86 & $2.17 \mathrm{E}-04$ \\
\hline Lipid metabolism & A2M/LRP1 & 10.40 & $2.67 \mathrm{E}-08$ \\
\hline Amino acid metabolism & TGM2 & -3.85 & $3.99 \mathrm{E}-08$ \\
\hline Negative regulation of HIF1A signaling & CITED2 & 3.61 & $5.16 \mathrm{E}-10$ \\
\hline Regulation of cell proliferation & IBP3 & 16.22 & $1.35 \mathrm{E}-10$ \\
\hline Apoptosis & NOXA & 729.95 & $7.75 \mathrm{E}-08$ \\
\hline Heme metabolism & FECH & 2.42 & $5.43 \mathrm{E}-09$ \\
\hline Other & MDR1 & -124.65 & $1.32 \mathrm{E}-11$ \\
\hline
\end{tabular}

Table 1. Differentially expressed genes in the "transcription: HIF-1 targets" pathway. List of transcripts DEG sorted by process and fold change, with respective p-value when compared PVEC vs. ABEC. 
immunomodulatory cellular communication network factor 2 (Ccn2/Ctgf, 16-fold), transforming growth factor beta 2 ( $T g f b 2$, ninefold) and $T g f b 3$ (33-fold) were higher, while $T g f b 1$ (threefold) was lower.

A substantial number of genes (32 of 47) included in the "FGF signaling in embryonic stem cell self-renewal and differentiation" pathway were differentially expressed in PVEC vs. ABEC. Amongst those, higher levels of genes encoding for secreted proteins noggin (Nog, 153-fold), insulin growth factor 2 (Igf2, 47-fold), gremlin 1 (Grem1, 30-fold) and Fgf2 predict PVEC propensity to inhibit Bone Morphogenetic Protein (BMP) signaling, and favor the maintenance of pluripotency and stemness in both neural and hematopoietic lineages ${ }^{18}$. Nonetheless, dramatically lower levels of the Sox 17 transcript (4222-fold) in PVEC likely precludes their transition into hematopoietic stem cells, thereby maintaining their commitment to the EC lineage ${ }^{19}$. Also, 31 of 46 genes associating with the "High shear stress-induced platelet activation" pathway were differentially expressed in PVEC vs. ABEC. There were significantly lower transcript levels of Von Willebrand factor ( $V w f$, 1066-fold), integrin beta 3 (Itgb3, 14-fold), and selectin platelet (Selp/P-selectin, 3556-fold), all of which were consistent with an immature, yet less thrombogenic and less inflammatory PVEC phenotype ${ }^{20}$.

Next, we analyzed canonical pathways after separating the dataset by DEG that were higher or lower in PVEC vs. ABEC. This identified the "role of activation of WNT signaling in progression of lung cancer" as the pathway with the greatest number of DEG whose expression was higher (36/77) in PVEC, and "immune response IFNa/ $\beta$ signaling via JAK/STAT" as the pathway with the greatest number of DEG whose expression was lower (25/64) in PVEC (Fig. 2d). Most of the DEG in the Wingless-Type MMTV Integration Site (WNT) pathway were increased by multiple fold (Table 2 and Supplementary Figure 2b). This included 10 of the WNT genes themselves (Wnt7a, 69-fold) as well as frizzled ligands of WNT, i.e. secreted frizzled-related protein 1 (Sfrp1, 500-fold) and frizzled class receptors ( $F z d 1,600$-fold; $F z d 3,300$-fold). This also included transcripts of the WNT complex associated proteins axin 1 (Axin1, 167-fold), Disheveled family members $D v l 1, D v l 2$ and $D v l 3$ (2-threefold), neuroprotective dickkopf WNT signaling pathway inhibitor 3 (Dkk3, 853-fold), and achaete-scute family bHLH transcription factor 1 (Ascl1/hASH1, 175-fold), a transcriptional regulator of many genes involved in neural development and differentiation. PVEC had also significantly higher transcript levels of lymphoid enhancer binding factor 1 (Lef-1, 64-fold), suggesting the engagement of $\beta$-catenin, downstream of WNT.

Genes associated with the Interferon $\alpha$ and $\beta$ (IFN $\alpha / \beta)$ Janus kinase/Signal Transducer and Activator of Transcription factors (JAK/STAT) signaling were significantly lower in PVEC vs. ABEC. These included signal transducer and activator of transcription (Stat) 4 (78-fold), Stat6 (eightfold), and Stat5 (fourfold), as well as the 2 key transducers of type I and type II IFN signaling, namely Stat1 (fourfold) and Stat2 (fourfold). Reduced expression of STAT1 is likely to uphold proliferative responses and stemness of neural progenitors of the subventricular (SVZ) niche ${ }^{21}$. Decreased IFN II signaling in PVEC was confirmed by lower transcript levels of STAT1 and STAT2 transcriptional targets, XIAP associated factor-1 (Xaf1, 232-fold), ubiquitin specific peptidase 18 (Usp18, 68-fold), interferon regulatory factor 7 (Irf7, 40-fold), caspase-8 (Casp8, fourfold), and interferon-induced protein with tetratricopeptide repeats 3 (Ifit3/RIG-G, threefold) ${ }^{22-24}$ (Supplementary Figure 2c).

The expression profile of SLC and $A B C$ transporters underscores differences in nutrient and metabolite transport between PVEC and ABEC. We checked whether expression levels of SLC differ between PVEC and ABEC. In mice, this family of membrane proteins includes 392 members that participate in brain development and in the formation and maintenance of the $\mathrm{BBB}^{25}$. Our results showed that 124 SLC transcripts were differentially expressed (61 higher and 63 lower) in PVEC vs. ABEC (Supplementary Figure 3a). Nineteen of the 61 that were higher in PVEC vs. ABEC increased by $>70$-fold (Table 3), including the glutamate transporters Slc1a3 (Eaat1/Glast1, 1466-fold), Slc1a2 (Eaat3, 70-fold), and Slc38a3 (Snat3, 131-fold) ${ }^{26}$, the mitochondrial ATP-Mg/Pi carrier Slc25a23 (237-fold) that protects neurons from glutamate toxicity ${ }^{27}$ and the zinc transporter Slc39a12 (141-fold), whose function is to support nervous system development ${ }^{28}$. This list also includes hormone transporters, such as Slcolc1 (Oatp14, 654-fold) and Slc16a2 (Mct8, 247-fold), which are involved in thyroid hormone (T4) transport and uptake in brain EC and neurons ${ }^{29}$, and the glucose transporters Slc2a3 (Glut3, 489-fold) and Slc2a13 (74-fold) that function to promote neural development and whose expression is enriched in the brain ${ }^{30}$. The relevance of the 63 SLC that were lower in PVEC vs. ABEC, including Slc9b1, Slc24a1, Slc46a3, Slc16a11, Slc6a3 (> 70-fold lower), is not totally clear and needs to be explored (Table 3).

Another well characterized superfamily of transporters in brain EC is the ATP-binding cassette (ABC) family, whose members participate in the BBB and the blood CSF barrier ${ }^{31}$. Eighteen out of $52 \mathrm{ABC}$ transporters were differentially expressed in PVEC vs. ABEC (Supplementary Figure 3b). Nine of those were significantly higher, including $A b c a 9$ (96-fold), $A b c a 8 a$ (eightfold), $A b c a 8 b$ (32-fold) and $A b c g 4$ (75-fold) that contribute to the transport and metabolism of lipid and cholesterol ${ }^{32}$. The other nine were significantly lower, including membrane P-glycoprotein ( $A b c b 1 a, 125$-fold and $A b c b 1 b$, sevenfold) whose expression at the luminal site of the $\mathrm{BBB}$ increases with gestational age to block the diffusion into the developing brain of deleterious substances and xenobiotics, marking the immature status of the BBB in PVEC ${ }^{33}$.

PVEC undergo a major transcriptome change upon co-culture with NPC. Since development of the radial glia/NPC is coordinated along the same spatiotemporal axis as the periventricular vascular plexus, we questioned whether the PVEC transcriptome qualitatively and/or quantitatively changes upon $48 \mathrm{~h}$ co-culture with NPC. ABEC co-cultured with NPC were used for comparison. Unsupervised principal component analysis (PCA) of the RNAseq data showed that cell type accounted for most of the variance between groups (PC1, 79.05\%). Also, ABEC/ABEC + NPC clustered closer than PVEC/PVEC + NPC along the PC2 axis (7.68\%), indicating a significantly greater impact of NPC co-culture on PVEC vs. ABEC transcriptomes (Fig. 3a).

Next, we performed supervised analysis to identify DEG in PVEC vs. ABEC monocultures or NPC cocultures. A total of 6241 genes were differentially expressed in PVEC vs. ABEC, including 5842 genes in PVEC 


\begin{tabular}{|c|c|c|c|}
\hline Family & Gene & Fold change & p-value \\
\hline \multirow{16}{*}{ Wingless-type MMTV integration site family } & Wnt7a & 69.05 & $9.66 \mathrm{E}-08$ \\
\hline & Wnt8b & 22.64 & $2.65 \mathrm{E}-08$ \\
\hline & Wnt2b & 13.46 & $3.66 \mathrm{E}-08$ \\
\hline & Wnt2b & 13.46 & $3.66 \mathrm{E}-08$ \\
\hline & Wnt7b & 12.13 & $5.17 \mathrm{E}-07$ \\
\hline & Wnt3 & 11.21 & $3.83 \mathrm{E}-08$ \\
\hline & Wnt3 & 11.21 & $3.83 \mathrm{E}-08$ \\
\hline & Wnt2 & 11.21 & $6.94 \mathrm{E}-05$ \\
\hline & Wnt2 & 11.21 & $6.94 \mathrm{E}-05$ \\
\hline & Wnt9a & 8.16 & $4.57 \mathrm{E}-10$ \\
\hline & Wnt9a & 8.16 & $4.57 \mathrm{E}-10$ \\
\hline & Wnt5b & 7.15 & $2.02 \mathrm{E}-07$ \\
\hline & Wnt5a & 5.34 & $5.90 \mathrm{E}-10$ \\
\hline & Wnt5a & 5.34 & $5.90 \mathrm{E}-10$ \\
\hline & Wnt4 & 2.37 & $6.04 \mathrm{E}-06$ \\
\hline & Wnt4 & 2.37 & $6.04 \mathrm{E}-06$ \\
\hline \multirow{7}{*}{ Frizzled } & Fzd1 & 599.47 & $6.89 \mathrm{E}-07$ \\
\hline & Fzd3 & 226.56 & $1.75 \mathrm{E}-08$ \\
\hline & Fzd2/10 & 78.70 & $1.80 \mathrm{E}-10$ \\
\hline & Fzd7 & 3.05 & $1.23 \mathrm{E}-07$ \\
\hline & Fzd8 & 2.69 & $5.28 \mathrm{E}-08$ \\
\hline & Fzd9 & -14.35 & $6.01 \mathrm{E}-10$ \\
\hline & Fzd4 & -18.05 & $7.14 \mathrm{E}-11$ \\
\hline \multirow{4}{*}{ Tcf(Lef) } & Lef1 & 63.82 & $2.17 \mathrm{E}-08$ \\
\hline & Tcf7 & 17.06 & $7.81 \mathrm{E}-09$ \\
\hline & Tcf7l2 & 2.03 & $7.89 \mathrm{E}-09$ \\
\hline & Tcf7l2 & 2.03 & $7.89 \mathrm{E}-09$ \\
\hline \multirow{3}{*}{ p38 MAPK } & Mapk13 & 25.61 & $1.40 \mathrm{E}-05$ \\
\hline & Mapk11 & -8.81 & $3.56 \mathrm{E}-09$ \\
\hline & Mapk12 & -16.20 & $7.54 \mathrm{E}-11$ \\
\hline \multirow{3}{*}{ Dishevelled segment polarity protein (DSH) } & Dvl3 & 2.95 & $1.08 \mathrm{E}-08$ \\
\hline & Dvl2 & 2.23 & $1.52 \mathrm{E}-07$ \\
\hline & Dvl1 & 2.01 & $4.97 \mathrm{E}-07$ \\
\hline \multirow{2}{*}{ Secreted frizzled-related protein } & Sfrp1 & 501.83 & $3.66 \mathrm{E}-08$ \\
\hline & Sfrp2 & 5.34 & $1.21 \mathrm{E}-06$ \\
\hline Dickkopf WNT signaling pathway inhibitor & Dkk3 & 853.00 & $1.07 \mathrm{E}-09$ \\
\hline Axin2 & Axin2 & 166.70 & $1.30 \mathrm{E}-08$ \\
\hline BMI-1 & Bmil & -2.35 & $1.46 \mathrm{E}-08$ \\
\hline Inhibitor of growth family & Ing4 & 2.21 & $5.33 \mathrm{E}-08$ \\
\hline \multirow{12}{*}{ Other } & hASH1 & 175.40 & $3.60 \mathrm{E}-10$ \\
\hline & NKD1 & 117.22 & $9.19 \mathrm{E}-07$ \\
\hline & ROR2 & 100.74 & $1.49 \mathrm{E}-08$ \\
\hline & WIF1 & 71.26 & $5.31 \mathrm{E}-08$ \\
\hline & NOTCH3 & 70.10 & $1.21 \mathrm{E}-08$ \\
\hline & VEGF-A & 10.14 & $1.15 \mathrm{E}-11$ \\
\hline & Porcn & 8.42 & $1.70 \mathrm{E}-07$ \\
\hline & Krm1 & 2.56 & $1.39 \mathrm{E}-08$ \\
\hline & $\mathrm{p} 21$ & 2.10 & $1.15 \mathrm{E}-08$ \\
\hline & c-Myc & -2.01 & $3.59 \mathrm{E}-07$ \\
\hline & \begin{tabular}{|l} 
Survivin \\
\end{tabular} & -2.38 & $4.90 \mathrm{E}-08$ \\
\hline & Oct-3/4 & -9.17 & $6.98 \mathrm{E}-04$ \\
\hline
\end{tabular}

Table 2. Differentially expressed genes involved in the WNT signaling pathway. List of transcripts DEG in the WNT pathway sorted by family/function, and its respective fold change and p-value when compared PVEC vs. ABEC. 


\begin{tabular}{|c|c|c|c|c|}
\hline Gene & Fold change & Protein name & Substrates & Family \\
\hline Slc1a3 & 1465.89 & EAAC1, EAAT1 & L-Glu, D/L-Asp & High-affinity glutamate and neutral amino acid transporter \\
\hline Slcolcl & 654.22 & OATP1C1 & T4, T3, rT3 & Organic anion transporter \\
\hline Slc2a3 & 489.48 & GLUT3 & Glucose, galactose, mannose, xylose & Facilitative GLUT transporter \\
\hline Slc4a3 & 295.16 & AE3 & Chloride bicarbonate & Bicarbonate transporter \\
\hline Slc6a15 & 280.17 & NTT73 & Large, neutral amino acids & $\begin{array}{l}\text { Sodium- and chloride-dependent neurotransmitter trans- } \\
\text { porter }\end{array}$ \\
\hline Slc16a9 & 247.31 & MCT9 & & Monocarboxylate transporter \\
\hline Slc25a23 & 236.53 & APC2 & ATP- $\mathrm{Mg}^{2+}, \mathrm{ATP}, \mathrm{ADP}, \mathrm{AMP}, \mathrm{Pi}$ & Mitochondrial carrier \\
\hline Slcola5 & 170.64 & OATP-3 & Taurocholate and thyroid hormones & Organic anion transporter \\
\hline Slc39a12 & 140.97 & ZIP12j, LZT-Hs8 & $\mathrm{Zn}$ & Metal ion transporter \\
\hline Slc16a2 & 131.42 & MCT8 & T2, rT3, T3, T4 & Monocarboxylate transporter \\
\hline Slc38a3 & 130.64 & SNAT3 & $\mathrm{Q}, \mathrm{H}, \mathrm{A}, \mathrm{N}$ & $\begin{array}{l}\text { System } A \text { and System } N \text { sodium-coupled neutral amino acid } \\
\text { transporter }\end{array}$ \\
\hline Slc4a4 & 108.59 & NBCe1 & Sodium bicarbonate (and/or carbonate) & Bicarbonate transporter \\
\hline Slc7a2 & 107.45 & CAT-2 (A or B) & Cationic L-amino acids & Cationic amino acid transporter/glycoprotein-associated \\
\hline Slc15a2 & 98.45 & PEPT2 & Di- and tri-peptides, protons, beta-lactam antibiotics & Proton oligopeptide cotransporter \\
\hline Slc6al & 76.36 & GAT-1 & GABA & $\begin{array}{l}\text { Sodium- and chloride-dependent neurotransmitter trans- } \\
\text { porter }\end{array}$ \\
\hline Slc6a17 & 73.98 & NTT4 & Neutral amino acids & $\begin{array}{l}\text { Sodium- and chloride-dependent neurotransmitter trans- } \\
\text { porter }\end{array}$ \\
\hline Slc2a13 & 73.70 & HMIT & Myo-inositol & Facilitative GLUT transporter \\
\hline Slc1a2 & 69.88 & EAAC1, EAAT3 & L-Glu, D/L-Asp & High-affinity glutamate and neutral amino acid transporter \\
\hline Slc24a3 & 69.45 & $\mathrm{Na}^{+} / \mathrm{K}^{+} / \mathrm{Ca}^{2+}$-exchange protein 3 & & $\mathrm{Na}^{+} /\left(\mathrm{Ca}^{2+}-\mathrm{K}^{+}\right)$exchange \\
\hline Slc22a18 & 0.0154 & ORCTL-2 & Probably organic anions & Organic cation/anion/zwitterion transporter \\
\hline Slc6a3 & 0.0124 & DAT & Dopamine & $\begin{array}{l}\text { Sodium- and chloride-dependent neurotransmitter trans- } \\
\text { porter }\end{array}$ \\
\hline Slc16a11 & 0.0106 & MCT11 & Pyruvate & Monocarboxylate transporter \\
\hline Slc46a3 & 0.0102 & & Lysosomal export of maytansine conjugates & Folate transporter family \\
\hline Slc24a1 & 0.0096 & NCKX1 & $\mathrm{Na}^{+}, \mathrm{Ca}^{2+}, \mathrm{K}^{+}$ & $\mathrm{Na}^{+} /\left(\mathrm{Ca}^{2+}-\mathrm{K}^{+}\right)$exchange \\
\hline Slc9b1 & 0.0015 & NHE1 & $\mathrm{Na}^{+}, \mathrm{Li}^{+}, \mathrm{H}^{+}, \mathrm{NH}^{4+}$ & $\mathrm{Na}^{+} / \mathrm{H}^{+}$exchanger \\
\hline
\end{tabular}

Table 3. Differentially expressed SLC transporters in PVEC vs. ABEC. List of SLC family transcripts whose expression was at least 70-fold higher or lower in PVEC vs. ABEC.

vs. ABEC monocultures, and 5712 in PVEC + NPC vs. ABEC + NPC. Of these genes, 529 were only differentially expressed in PVEC vs. ABEC at baseline, with 457 being exclusive to this comparison. On the other hand, 399 genes were only differentially expressed in PVEC + NPC vs. ABEC + NPC co-cultures, with 337 being exclusive to this comparison. Notably, 5313 genes were differentially expressed regardless of the culture condition, with 5053 being exclusively differentially expressed in PVEC vs. ABEC and PVEC + NPC vs. ABEC + NPC (Fig. 3b).

NPC co-culture resulted in robust changes in PVEC but not in ABEC transcriptome. There were 413 DEG in PVEC vs. PVEC + NPC, 224 were upregulated and 189 were downregulated. In contrast, just 15 genes were differentially expressed in ABEC vs. ABEC + NPC, 6 were upregulated and 9 were downregulated. Only 2 of the genes that were differentially expressed upon co-culture with NPC were common to PVEC and ABEC: lysophosphatidylcholine acyltransferase 2 (Lpcat2), was differentially expressed across all comparison groups (30-fold higher in PVEC vs. ABEC and in PVEC + NPC vs. ABEC + NPC, and threefold higher in PVEC + NPC vs. PVEC and in $\mathrm{ABEC}+\mathrm{NPC}$ vs. ABEC) and synuclein alpha (Snca) was only differentially expressed when PVEC or ABEC were co-cultured with NPC (threefold higher in ABEC + NPC vs. ABEC and 2.5-fold higher in PVEC + NPC vs. PVEC) (Fig. 3b). Glucosaminyl (N-acetyl) transferase 1 (Gcnt1) was the only gene whose expression was exclusively changed in ABEC + NPC vs. ABEC (twofold higher). On the other hand, 30 genes were exclusively modified in PVEC + NPC vs. PVEC, including regulators of cell proliferation, such as the BCL2/adenovirus E1B interacting protein 3 (Bnip3) and cyclin dependent kinase inhibitor 1C (P57) (Cdkn1c), and SLC transporters such as the thiamine transporter Slc35f3, and the sodium bicarbonate co-transporter Slc4a10 that regulates neuronal pH in the choroid plexus, all of which were upregulated by twofold to threefold after co-culture.

Of the 413 genes that were differentially expressed in PVEC vs. PVEC + NPC, 321 were also differentially expressed in PVEC vs. ABEC, as gauged by supervised analysis. Using the self-organizing map (SOM) unsupervised algorithm, we clustered these genes into patterns based on their expression profile in PVEC vs. ABEC monocultures, and on their directional change upon co-culture with NPC. Patterns I-IV include genes that were upregulated in PVEC upon NPC co-culture. In patterns I-III, this upregulation further amplified the physiologic differences between PVEC vs. ABEC (Fig. 3c), whereas in pattern IV it brought PVEC expression levels closer to that of ABEC. GO_bp enrichment analysis of these clusters, followed by data summarizing using REVIGO, identified biologic processes that were enriched in each pattern. These included "Regulation of anatomical structure size" in pattern I (Fig. 3d), and "Regulation of cellular component organization" in pattern II. Genes clustered in Patterns III and IV did not enrich for any specific GO_bp. Patterns V, VI and VII include genes 
a

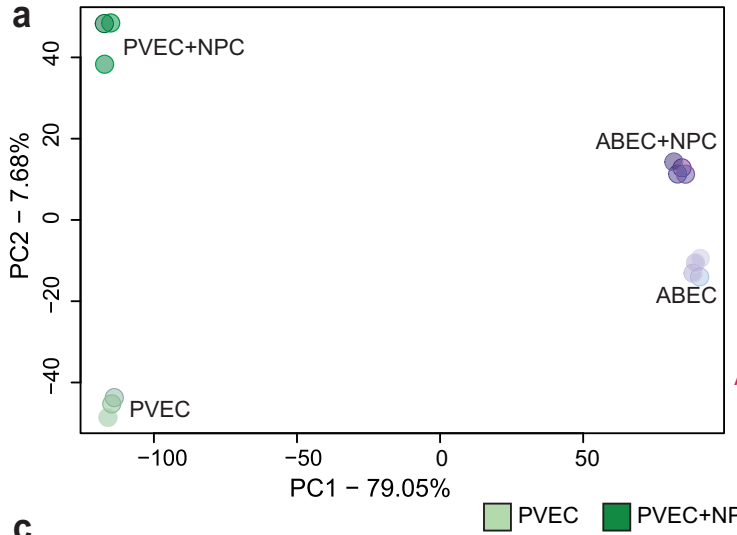

C b

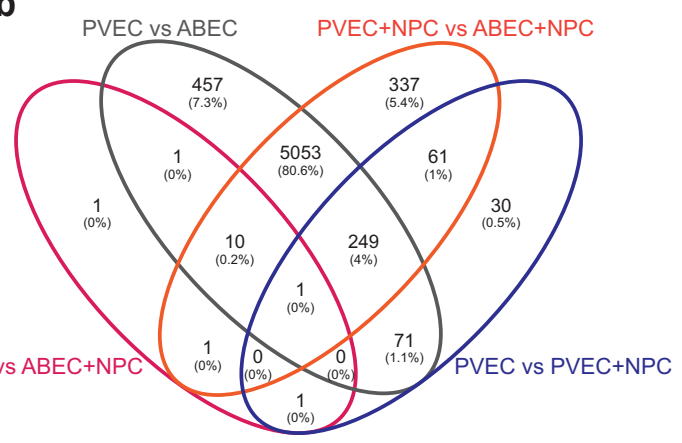

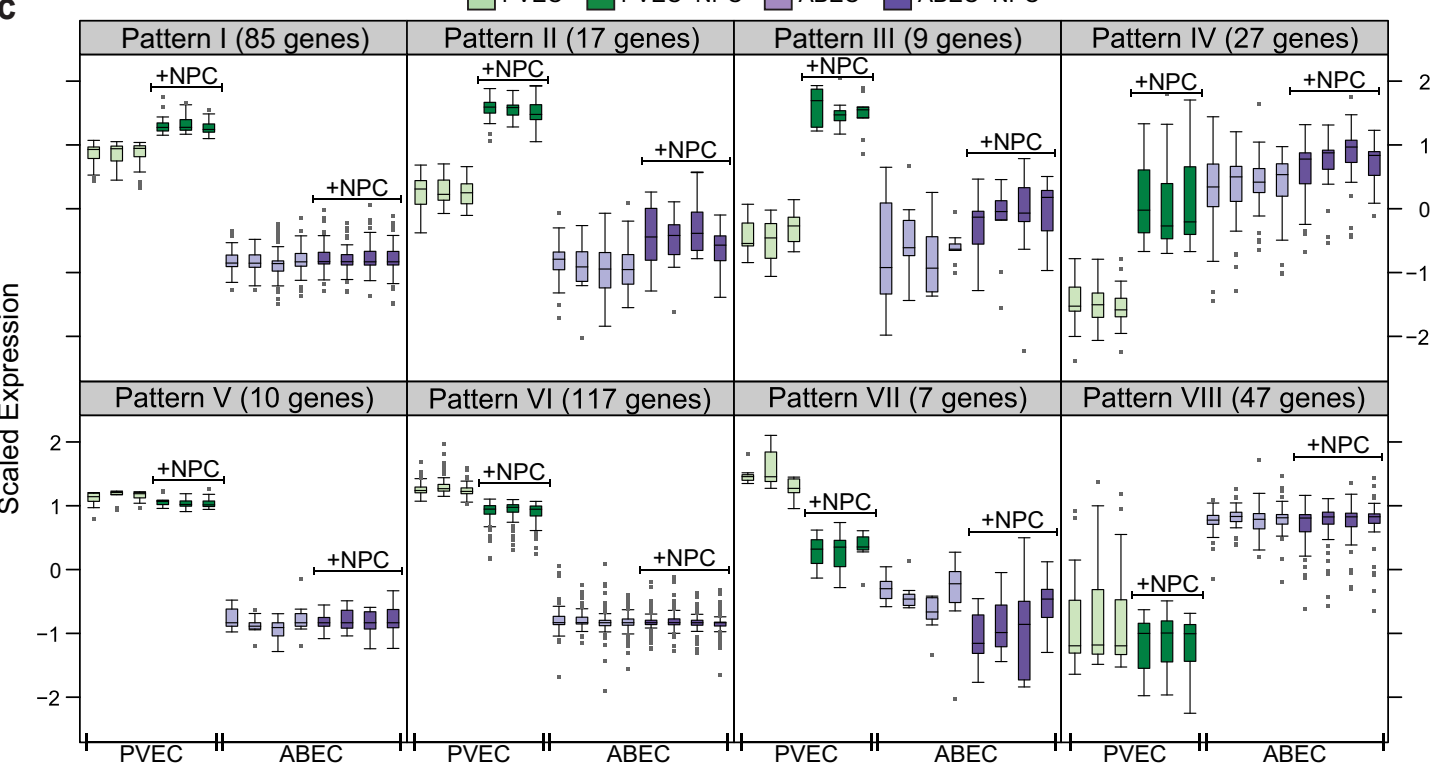

d

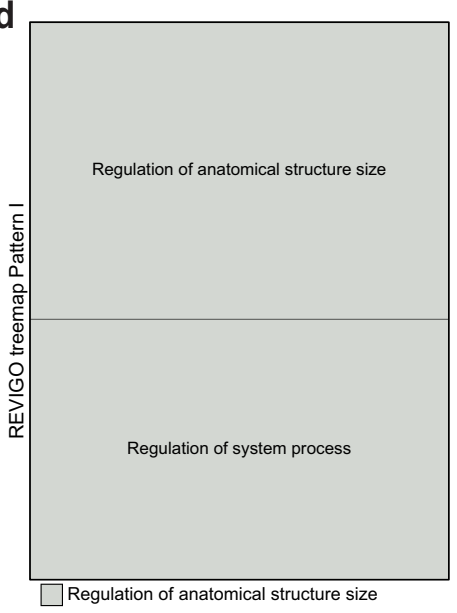

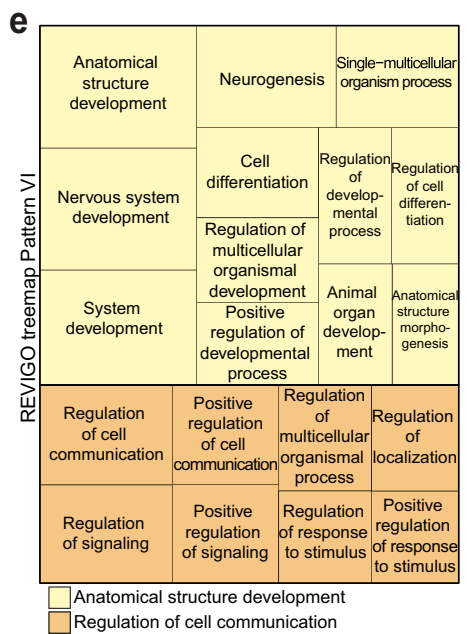

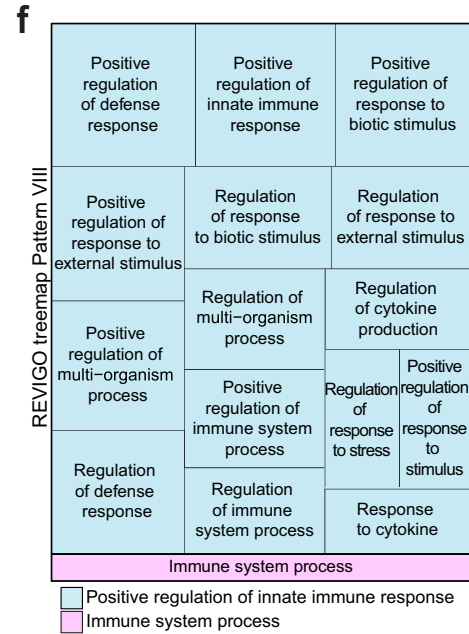

Figure 3. Comparative transcriptomic analysis of PVEC vs. ABEC following co-culture with NPC indicate major changes in PVEC not ABEC. (a) Principal component analysis (PCA) of transcriptome. Colors of circles refer to the origin of samples: green PVEC and purple ABEC, light versus dark shades indicate mono vs. NPC co-culture conditions. (b) Venn Diagram of DEG in PVEC vs. ABEC (grey ellipse), PVEC + NPC vs. ABEC + NPC (orange ellipse), ABEC vs. ABEC + NPC (pink ellipse) and PVEC vs. PVEC + NPC (blue ellipse). (c) Eight different self-organizing maps (SOM) patterns of gene expression profiles were identified amongst the 321 genes that were different in PVEC vs. PVEC + NPC and also in PVEC vs. ABEC. The upper panels display patterns whose gene expression is upregulated in PVEC + NPC as compared to baseline PVEC. Data is shown as standard boxplots (box and whisker plots), depicting the median bar and the dots representing the outliers. Lower panels depict patterns whose gene expression is downregulated in PVEC+ NPC as compared to baseline PVEC. Treemap representation of GO_bp (biological process) using REVIGO (reduced visualization of GO) corresponding to (d) Pattern I, (e) Pattern VI and (f) Pattern VIII. 


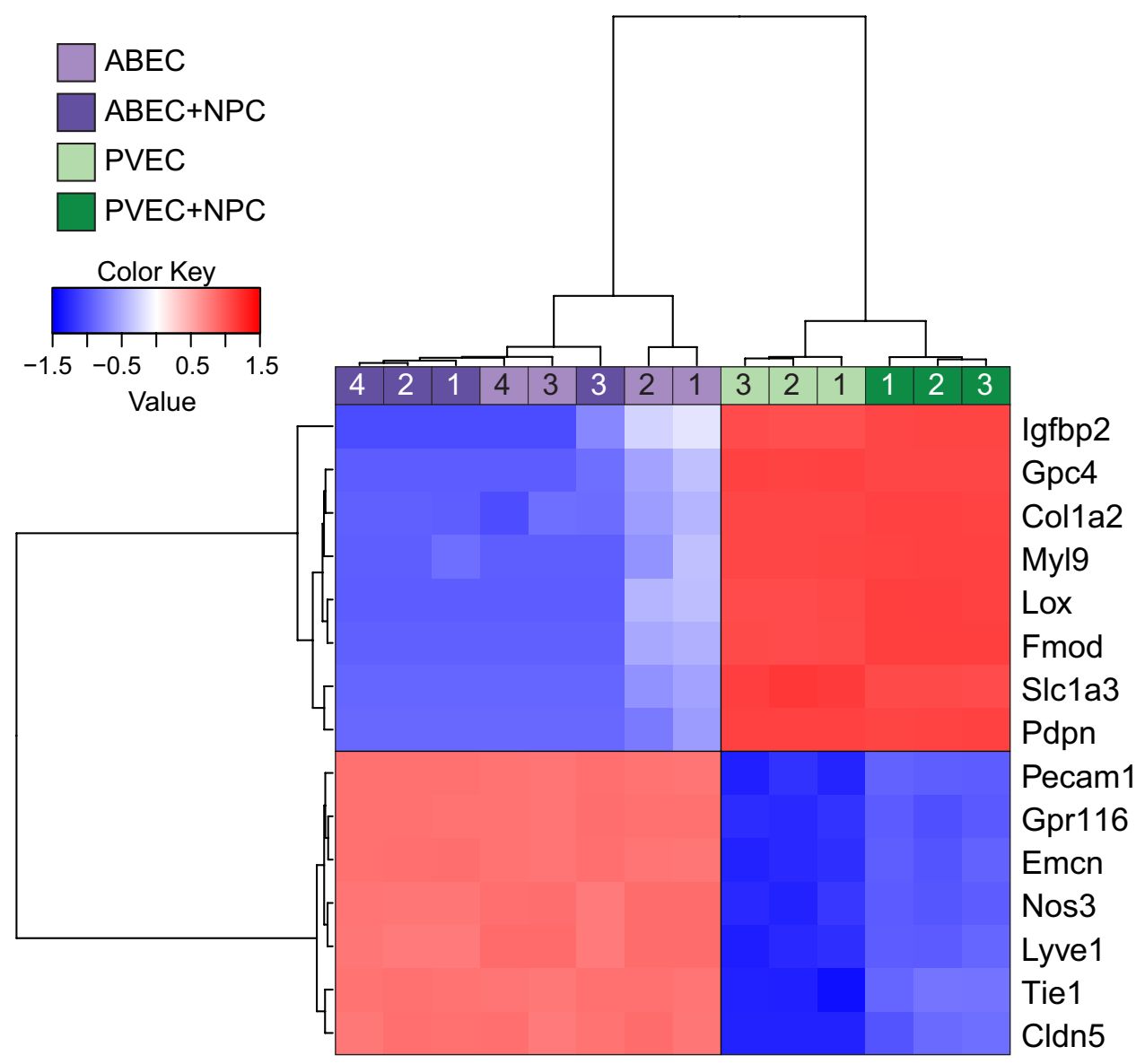

Figure 4. PVEC exhibit a distinct gene signature independent from culture condition. Hierarchical clustering and heatmap analysis of the top 15 DEG in PVEC $(n=6)$ vs. ABEC $(n=8)$. Gene expression is shown in a pseudocolor scale. Red indicates genes with higher expression and blue indicates genes with lower expression in PVEC vs. ABEC.

whose basal expression levels were higher in PVEC vs. ABEC monocultures and were downregulated upon NPC co-culture to levels closer to that of ABEC. GO_bp enrichment analysis, followed by data summarizing using REVIGO of Pattern VI showed an enrichment for "Anatomical structure development" and "Regulation of cell communication" (Fig. 3e). Pattern VIII encompass genes whose expression was initially lower in PVEC vs. ABEC monocultures and slightly increased upon NPC co-culture, albeit remaining lower than ABEC's levels. By GO_bp analysis and REVIGO summarizing, Pattern VIII enriched for "Positive regulation of innate immune response" and "Immune system process" (Fig. 3f).

The top differentially expressed genes in PVEC, regardless of culture condition, capture the transcriptional signature of these cells. Heatmap analysis of the top DEG regardless of mono or coculture conditions-proposed as the transcriptional signature of PVEC-is shown in Fig. 4. The most upregulated genes in PVEC/PVEC + NPC vs. ABEC/ABEC + NPC were insulin-like growth factor binding protein 2 (Igfbp2), Lox, fibromodulin (Fmod), Myl9, Gpc4, collagen, type I, alpha 2 (Col1a2), Slc1a3 and Pdpn; the most downregulated genes were Pecam1, Tie1, Nos3, Gpr116/Adgrf5, Emcn, Lyve1 and Cldn5. In addition to previously discussed EC markers (Pecam1, Tie1 and Nos3), Gpr116/Adgrf5, an adhesion molecule that is also broadly expressed in EC, was 10,000-fold lower in PVEC/PVEC + NPC vs. ABEC/ABEC + NPC. The importance of Gpr116/Adgrf5 expression in the brain endothelium is emphasized by the brain vascular leakage that occurs in Gpr116/Adgrf5 EC-specific knockout mice ${ }^{34}$. Hierarchical clustering of the samples showed distinct separation between PVEC and PVEC+ NPC. In contrast, ABEC vs. ABEC+ NPC did not separate well (Fig. 4). Heatmap analysis of the data strongly corroborates the specific transcriptional signature associated with the developmental stage of PVEC.

Pathway analysis of PVEC co-cultured with NPC ascertains an overall decrease in complement and increase in WNT/ $\beta$-catenin related transcripts. In order to better understand the effect of NPC co-culture on PVEC, we performed canonical pathway enrichment analysis on DEG. Among the top ten most modified pathways, five were related to the immune system response/complement, namely classical complement (16 genes modified in our dataset out of 53 in database), lectin-induced complement (15 out of 50), and alterna- 


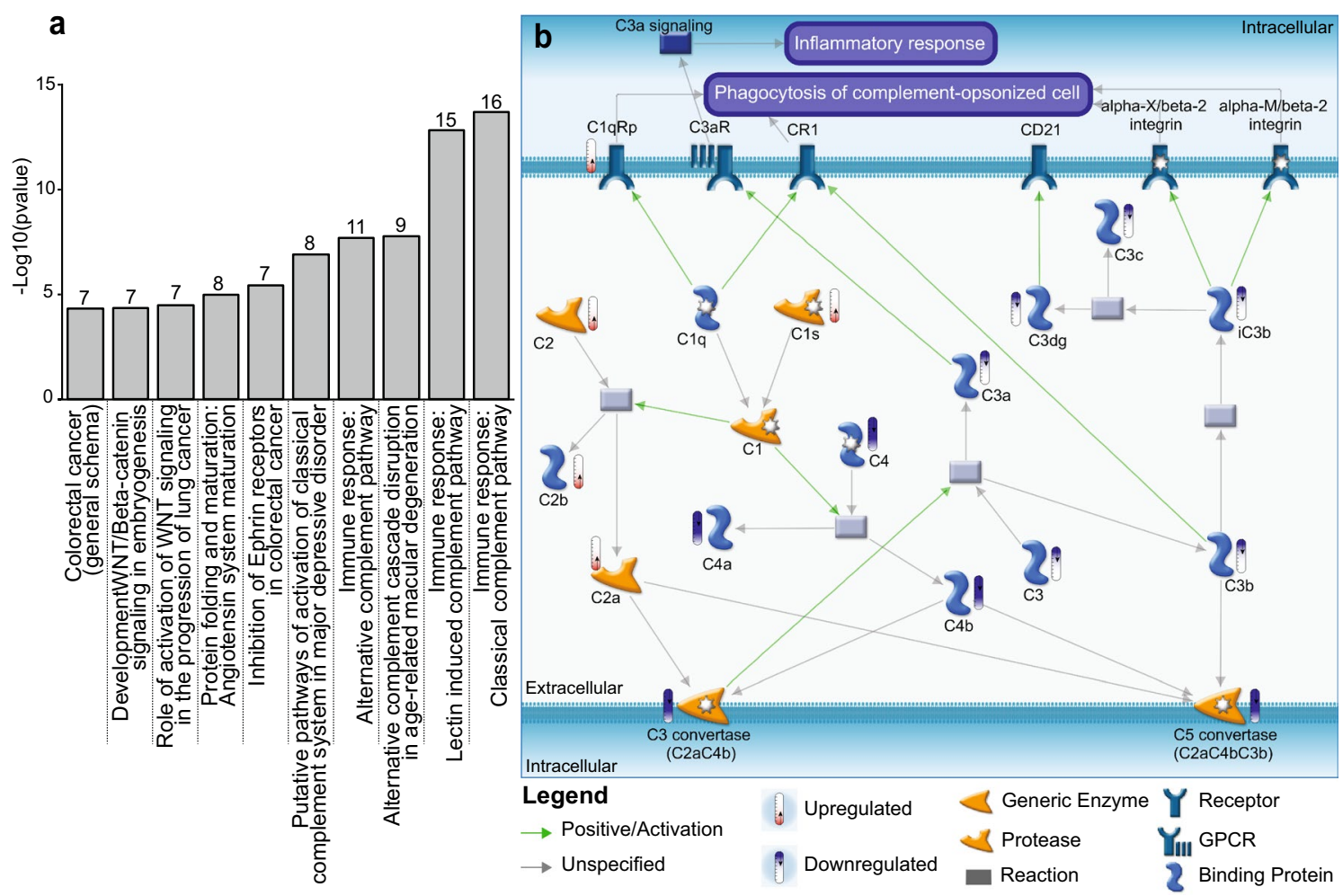

Figure 5. Canonical pathway enrichment analysis identifies immune response classical complement pathway as the ones most enriched in PVEC co-cultured with NPC. (a) Canonical pathway enrichment using the Metacore from Clarivate Analytics identified the top 10 most enriched pathways in PVEC vs. PVEC + NPC. (b) Depiction of the top most modified pathway, "Immune response: Classical complement pathway". The legend included in the figure decodes the colored shapes and arrows used in the picture. Colored bars close to each protein name indicates whether the DEG was upregulated (red) or down-regulated (blue) upon co-culture of PVEC with NPC.

tive complement (9 out of 31) (Fig. 5a). Remarkably, the pathway with highest enrichment score in PVEC + NPC i.e. "Immune response: Classical complement pathway" included key components of the complement system whose transcript levels were significantly decreased upon co-culture. For instance, a decrease in complement component 3 (C3, 4.7-fold) and C4 (31.5-fold) implies lower levels of the C3 and C5 convertase complexes (Fig. 5b). Similar to our baseline PVEC vs. ABEC data, 2 of the 10 enriched pathways were related to WNT signaling, namely the "role of activation of WNT in the progression of lung cancer" (7 out of 41), and "development: WNT/ $\beta$ catenin signaling in embryogenesis" (7 of 43) (Fig. 5a).

Network analysis emphasizes the maintenance of stemness as a feature of PVEC. We previously reported that co-culturing PVEC with NPC delays expression of $\beta$-tubulin, a marker of stemness in NPC ${ }^{4}$. To better characterize this observation, we performed a network analysis of PVEC vs. PVEC + NPC transcriptomes and identified 'Oct-3/4, NANOG, Neuronatin, TRIL' as one of the top modulated networks (Supplementary Figure 4). A 100-fold increase in mRNA levels of Pou $5 \mathrm{fl}$ that encodes for Oct-3/4, a transcription factor that promotes pluripotency and self-renewal of undifferentiated cells $\mathrm{s}^{35}$, was the most salient change in this network. Also, an increase in the transcription factor Nanog (2.4-fold) and a decrease in neuronatin (Nnat, twofold), that directs cells into the neural lineage ${ }^{36}$, strongly validates our proposed hypothesis-PVEC and NPC cross-talk to maintain their respective early-stage of differentiation. In addition to influencing stemness, higher Oct $3 / 4$ transcripts in PVEC co-culture were associated with 2-fourfold lower levels of pro-inflammatory TLR4 interactor with leucine-rich repeats (Tril). Tril is highly expressed in the brain, specifically in glial cells where its knockdown attenuates cytokine production ${ }^{37}$. These latter results further highlight the combined anti-inflammatory and maintenance of stemness phenotype of PVEC.

\section{Discussion}

We previously showed that one of the functions of PVEC is to maintain the proliferative capacity and the progenitor status of $\mathrm{NPC}^{4}$. To better characterize these cells, we compared their basal transcriptome to that of adult brain EC. Our data demonstrate that PVEC and ABEC transcriptomes are strikingly distinct at baseline (>5000 genes), reflecting the mature state of ABEC and their barrier properties (high expression of Tie1, Pecam1, Nos3, and Cldn5) vs. the immature state of PVEC. PVEC immaturity is evidenced by their expression of genes usually associated with periventricular NPC, namely S1pr3, Igfb2, Igfbp5, Slc1a3 and Gpc4 ${ }^{31,38-40}$. SIPR3 drives 
S1P-induced proliferation of NPC ${ }^{38}$, IGFBP2 and IGFBP5 promote NPC proliferation and self-renewal, whilst precluding their differentiation ${ }^{39}$, and Slc1a3 contributes to NPC proliferation through glutamate transport ${ }^{41}$. Heparan sulphate proteoglycan, Glypican4 (GPC4) modulates FGF signal transduction, which is required for early dorsoventral forebrain patterning ${ }^{40}$. This agrees with $F g f 2$ transcripts being significantly higher in PVEC vs. ABEC.

PVEC also expressed higher levels of glucose transporters (Slc2a3 and Slc2a13) and glutamate transporters (Slc1a3, Slc1a2, Slc1a6 and Slc38a3). The brain receives about $20 \%$ of total body glucose and uses $80 \%$ of this energy to maintain the homeostasis of the glutamate/GABA glutamine cycle. While glutamate is important for synaptic function, its extracellular levels must be tightly controlled to avoid excitotoxicity ${ }^{42}$. Lack of the brainspecific Slc2a3 (Glut3) has profound effects on neural development ${ }^{30}$. Accordingly, its heightened expression in PVEC highlights the unique ability of these cells to promote brain development. The abundance of glutamate transporters in PVEC may have different implications, either to provide the substrate for NPC proliferation and synthesize GABA to generate GABAergic neurons, or to increase the brain-to-blood efflux of glutamate (Slc38a3) in order to limit excitotoxicity.

ABC transporters, including Abca9, Abca8a, Abca8b and Abcg4, all involved in lipid transport and metabolism were also higher in PVEC. This could be an important feature of PVEC since regulation of cholesterol homeostasis in the CNS relies on de novo cholesterol synthesis and efficient lipid transport and recycling within the brain $^{43}$. Consistent with the latter, PVEC also expressed significantly higher levels of Mfsd2a (147-fold) than ABEC, an omega- 3 fatty acid transporter that is essential for brain development ${ }^{44}$.

Other genes whose expression was higher in PVEC, namely Lox, Fmod and Colla2, are involved in the formation of ECM. The extracellular enzyme Lysyl oxidase (LOX), that deaminates peptidyl lysyl residues to promote cross-linking of fibrillar collagens and elastin, activates AKT in EC to increase VEGF expression and promote angiogenesis ${ }^{45}$. This agrees with significantly higher Vegf transcripts in PVEC vs. ABEC. The ECM protein Fibromodulin (FMOD) participates in the assembly of collagen fibers. Since overexpression of FMOD can reprogram cells to express pluripotency markers ${ }^{46}$, we surmise that its heightened expression in PVEC maintains the neurovascular niche. Furthermore, Collagen I triggers EC to assume a spindle-shaped morphology and to align into solid cord-like structures that resemble the pre-capillary cords of embryonic angiogenesis ${ }^{47}$. Hence, higher levels of Collagen I in PVEC is in line with these cells' ability to regulate angiogenesis and neurovascular patterning in the embryonic brain.

By GO analysis of DEG in PVEC vs. ABEC. "Nervous system development" and related categories were the most enriched, mostly with genes that were higher in PVEC vs. ABEC. This result concurs with neural development as the dominant program that characterizes this key function of PVEC.

On analyzing differentially expressed pathways, HIF-1A topped the list. This corresponds with embryo development occurring in a hypoxic environment where many of the HIF-1A targets are coopted to fulfill the requirements of a rapidly growing brain ${ }^{48}$. Although Hif-1 a mRNA levels were lower in PVEC vs. ABEC, transcript levels of many of its direct target genes were significantly higher, suggesting its activity was increased in these cells. For example, Cxcr4, a HIF-1A target gene expressed in rapidly dividing Nestin positive NPC, had its mRNA levels $>400$-fold higher in PVEC vs. ABEC ${ }^{49}$, so were transcript levels of the HIF1-A pro-angiogenic targets Vegfa, Fgf2, Thrombospondin1, and Angiopoietin $2^{50}$.

When we restricted our analysis to DEG that were significantly higher in PVEC, the top enriched pathway was WNT signaling. WNT/ $\beta$-catenin signaling prevents premature differentiation of NPC to neurons ${ }^{51}$. Higher expression of members of this pathway in PVEC corroborates our previous results showing that PVEC, but not ABEC, delay NPC differentiation ${ }^{4}$. Indeed, expression levels of the LEF-1 transcription factor, a positive regulator of NPC proliferation, was 64-fold higher in PVEC vs. ABEC. WNT signaling also positively regulates angiogenesis in the brain and contributes to BBB formation and integrity ${ }^{52,53}$. WNT ligands are expressed by NPC in the ventricular region during the same epoch as cerebral angiogenesis. Notably, transcript levels of the WNT ligands, $W n t 7 a$ and $W n t 7 b$, and of their receptors (Frizzled) were significantly higher in PVEC vs. ABEC. This suggests that PVEC act in an autocrine and paracrine manner to maintain the proliferative status of the NVU.

When we restricted our analysis to DEG that were significantly lower in PVEC, the top enriched pathway was "Immune response IFNa/ $\beta$ signaling via JAK/STAT", a novel observation during brain development that dovetails with data indicating that mounting an antiviral response causes differentiation of pluripotent stem cells ${ }^{54}$. Immune activation and secondary upregulation of chemokines and cytokines negatively regulate NPC proliferation and drives their differentiation into the neuronal and astroglial lineages in a STAT-dependent manner. Our results show that 5 of the 6 STAT, i.e. Stat1, 2, 4, 5 and 6 were significantly lower in PVEC vs. ABEC. This is in keeping with expression levels of most these STAT being characteristically low $(1,4$ and 5) or even undetectable (STAT6) in the embryonic mouse brain ${ }^{55,56}$. Importantly, transcript levels of a number of downstream targets of these STATs were also significantly lower in PVEC vs. ABEC. These include pro-apoptotic XAF1 and Caspase- $8^{57,58}$, RIG-G, a key mediator of the IFNa/ $\beta$ anti-proliferative effect ${ }^{59}$, and IRF7 that drives stem cells to mesodermal differentiation. In fact, expression of the pluripotency genes KLF4, SOX2 and OCT4 is totally irreconcilable with the transcriptional program of IRF $7^{54}$. Altogether, reduced expression of these 4 genes in PVEC showcases their stemness, proliferative capacity, and resistance to apoptotic stimuli. Stat 3 , is the only STAT member whose expression is threefold higher in PVEC vs. ABEC. STAT3 activation maintains the undifferentiated state of SVZ progenitors ${ }^{60}$ while its loss decreases the number of Nestin positive NPCs in the brain $^{61}$. Consequently, higher levels of STAT3 in PVEC serves the same purpose as lower expression of the other STATs i.e. maintaining stemness and boosting proliferation.

Although differences between PVEC and ABEC transcriptomes at baseline far exceeded any effects of NPC coculture, the PVEC, but not the ABEC, transcriptome still significantly changed by co-culture (413 vs. 15 genes), highlighting their unique partnership with NPC. Decreased expression of complement immune response genes was exclusively noted in PVEC co-cultured with NPC. This is a novel observation whose biologic consequences 
still need to be investigated. The only known non-immune function of complement in the brain is to regulate normal postnatal synaptic pruning and brain wiring ${ }^{62}$.

In conclusion, our data characterizes for the first time the gene signature of PVEC and their distinct partnership with NPC. The identity of these cells underpins their unique role in promoting brain development and maintaining a low inflammatory environment that supports a healthy neurovascular niche. Although we acknowledge that this study may still be limited by some changes that may occur following EC culturing and serial passaging, it offers a road map for additional studies aimed at expanding PVEC characterization by analyzing their transcriptome in their anatomic context and exploring their neuro-regenerative potential in different brain pathologies.

\section{Materials and methods}

Ethical consideration. All mice use was in strict compliance with current United States government regulations concerning the care and use of laboratory animals. Animal protocols were approved by the Beth Israel Deaconess Medical Center Institutional Animal Care and Use Committee. All mice were anesthetized with isoflurane during procedures and provided with appropriate pain relief.

Cell lines. Mouse primary ABEC derived from C57BL/6 were obtained from CellBiologics (Chicago, IL) and grown in DMEM-KO medium supplemented with 10\% fetal bovine serum (FBS), 10\% horse serum, $100 \mu \mathrm{g}$ Endothelial Mitogen (Biomedical Technologies, Stoughton, MA), 4 mM L-Glutamine and 1\% penicillin/streptomycin.

The neural progenitor NE-4C cell line (NPC) was obtained from American Type Culture Collection (Manassas, VA), and expanded for use in this study. This cell line maintains progenitor status, as evaluated by specific markers, after multiple passages ${ }^{2}$. NE-4 cells were cultured in DMEM-KO media supplemented with $10 \%$ FBS, $4 \mathrm{mM}$ L-Glutamine, $1 \%$ Penicillin/Streptomycin.

Isolation of PVEC from embryonic mice brain. At E15-16 the pial vessels from male and female C57BL/6 mice (Charles River Laboratories, Wilmington, MA) were removed ${ }^{63}$. The remaining telencephalon brain containing the periventricular vessels was dissociated into single cells as described ${ }^{4}$, and plated on a Type I rat tail collagen (BD Bioscience, San Jose, CA) coated flask containing DMEM-KO medium with 10\% FBS (Fisher Scientific, Pittsburgh, PA), 10\% Horse Serum, $100 \mu \mathrm{g}$ Endothelial Mitogen (Biomedical Technologies, Stoughton, MA), $4 \mathrm{mM}$ L-Glutamine, and 1\% Penicillin/Streptomycin. After approximately 5 days, endothelial cells were purified using the EasySep mouse phycoerythrin (PE) positive selection kit conjugated to CD31 (Stemcell Tech, Vancouver, Canada). Cells labeled with anti-CD31, were bound to dextran coated magnetic particles by tetrameric antibody complexes recognizing PE and dextran, as per the manufacturer recommendations. Only those CD31 positive cells that bound to the magnetic beads were further cultured and used at passage 5 in all subsequent experiments. Flow cytometry was used to confirm endothelial-specificity using bona fide EC markers as described ${ }^{4}$ (Supplementary material and methods and Supplementary Figure 1).

RNA extraction. In order to compare baseline gene expression between PVEC and ABEC, cells were grown as monocultures for $48 \mathrm{~h}$ before RNA extraction using the RNeasy Mini Kit (Qiagen, Germantown, MD). To understand the NPC effect on global gene expression, PVEC and ABEC $\left(3 \times 10^{4} \mathrm{EC} / \mathrm{cm}^{2}\right)$ were seeded in the lower chamber of a 24-transwell plate and $1 \times 10^{4} \mathrm{NPC} / \mathrm{cm}^{2}$ were added to the upper chamber. We kept the coculture for $48 \mathrm{~h}$ in $0.1 \mathrm{~mL}$ and $0.5 \mathrm{~mL}$ complete medium in the upper and lower chambers, respectively. After this time, RNA was extracted from the EC seeded in the lower chamber.

Transcriptional profiling. Double-stranded cDNA sequencing libraries were generated using the Illumina TruSeq kit per the manufacturer's protocol. High quality libraries were sequenced on an Illumina HiSeq 2000 (Illumina, Inc., San Diego, CA). To achieve comprehensive coverage for each sample, we generated 30-35 million paired end reads. Raw sequencing was pre-processed, quality checked, aligned to mouse genome, and unique numbers of reads counted. The read count-based expression data was normalized using the voom approach that estimates the mean-variance relationship of the log-transformed transcript counts data to generate a precision weight for each expressed transcript ${ }^{64}$. Differentially expressed transcripts were identified from the normalized dataset using Metaboanalyst and Metacore analysis workflow (Clarivate Analytics ver. 6.11, build 41105, GeneGo, Thomson Reuters, USA), based on absolute fold change (FC) and multiple test-corrected p-values based on FDR. Transcripts were considered significantly differentially expressed if p-values were $<0.05$ and absolute fold change (FC) was $>2$.

Self organizing map (SOM) clustering. We used the SOM clustering technique to identify groupdependent patterns of DEG in PVEC vs ABEC and PVEC vs PVEC + NPC ${ }^{65}$. SOM allows the grouping of gene expression patterns into an imposed structure in which adjacent clusters are related, thereby identifying sets of samples that follow certain expression patterns across groups. We performed sample-based SOM clustering (som package in R) using Pearson correlation coefficient-based distance metrics, which resulted in six sample clusters with distinct expression profiles.

Statistical analysis. Unsupervised analysis. Principal component analysis (PCA) and hierarchical clustering were used to perform unsupervised analysis on normalized and preprocessed data. Analysis were made in $\mathrm{R}$ and GenePattern ${ }^{66}$. 
Supervised analysis. MetaCore from Clarivate Analytics was used to perform enrichment analysis in order to determine the functional significance of DEG. We identified the top modified pathways and networks in PVEC vs. ABEC, PVEC + NPC vs. ABEC+NPC, PVEC vs. PVEC + NPC and ABEC vs. ABEC+NPC. The GO_bp results were subjected to a REduce and Visualize Gene Ontology tool (REVIGO) ${ }^{67}$.

Received: 17 July 2020; Accepted: 2 November 2020

Published online: 23 November 2020

\section{References}

1. Wittko-Schneider, I. M., Schneider, F. T. \& Plate, K. H. Cerebral angiogenesis during development: Who is conducting the orchestra? In Cerebral Angiogenesis Vol. 1135 (ed. Milner, R.) 3-20 (Springer, New York, 2014).

2. Vasudevan, A., Long, J. E., Crandall, J. E., Rubenstein, J. L. R. \& Bhide, P. G. Compartment-specific transcription factors orchestrate angiogenesis gradients in the embryonic brain. Nat. Neurosci. 11, 429-439 (2008).

3. Carmeliet, P. Blood vessels and nerves: Common signals, pathways and diseases. Nat. Rev. Genet. 4, 710-720 (2003).

4. Vissapragada, R. et al. Bidirectional crosstalk between periventricular endothelial cells and neural progenitor cells promotes the formation of a neurovascular unit. Brain Res. 1565, 8-17 (2014).

5. Guo, S. \& Lo, E. H. Dysfunctional cell-cell signaling in the neurovascular unit as a paradigm for central nervous system disease. Stroke 40, S4-S7 (2009).

6. Guo, S. et al. The vasculome of the mouse brain. PLoS ONE 7, e52665 (2012).

7. Segura, I., De Smet, F., Hohensinner, P. J., de Almodovar, C. R. \& Carmeliet, P. The neurovascular link in health and disease: An update. Trends Mol. Med. 15, 439-451 (2009).

8. Chen, Z.-Y., Asavaritikrai, P., Prchal, J. T. \& Noguchi, C. T. Endogenous erythropoietin signaling is required for normal neural progenitor cell proliferation. J. Biol. Chem. 282, 25875-25883 (2007).

9. Lok, J. et al. Cell-cell signaling in the neurovascular unit. Neurochem. Res. 32, 2032-2045 (2007).

10. Neuwelt, E. A. Mechanisms of disease: The blood-brain barrier. Neurosurgery 54, 131-140 (2004). (discussion 141-142).

11. Urich, E., Lazic, S. E., Molnos, J., Wells, I. \& Freskgård, P.-O. Transcriptional profiling of human brain endothelial cells reveals key properties crucial for predictive in vitro blood-brain barrier models. PLoS ONE 7, e38149 (2012).

12. Muffley, L. A. et al. Differentiation state determines neural effects on microvascular endothelial cells. Exp. Cell Res. 318, 2085-2093 (2012).

13. Rauch, M. F., Michaud, M., Xu, H., Madri, J. A. \& Lavik, E. B. Co-culture of primary neural progenitor and endothelial cells in a macroporous gel promotes stable vascular networks in vivo. J. Biomater. Sci. Polym. Ed. 19, 1469-1485 (2008).

14. Astarita, J. L., Acton, S. E. \& Turley, S. J. Podoplanin: Emerging functions in development, the immune system, and cancer. Front. Immunol. 3, 283 (2012).

15. Bondy, C. \& Lee, W. H. Correlation between insulin-like growth factor (IGF)-binding protein 5 and IGF-I gene expression during brain development. J. Neurosci. 13, 5092-5104 (1993).

16. Fico, A. et al. Modulating glypican4 suppresses tumorigenicity of embryonic stem cells while preserving self-renewal and pluripotency. Stem Cells 30, 1863-1874 (2012).

17. Goncharov, N. V., Nadeev, A. D., Jenkins, R. O. \& Avdonin, P. V. Markers and biomarkers of endothelium: When something is rotten in the state. Oxid. Med. Cell. Longev. 2017, 1-27 (2017).

18. LaVaute, T. M. et al. Regulation of neural specification from human embryonic stem cells by BMP and FGF. Stem Cells 27, 1741-1749 (2009).

19. Clarke, R. L. et al. The expression of Sox17 identifies and regulates haemogenic endothelium. Nat. Cell Biol. 15, 502-510 (2013).

20. Rusu, L. \& Minshall, R. D. Endothelial cell von Willebrand factor secretion in health and cardiovascular disease. In Endothelial Dysfunction-Old Concepts and New Challenges (ed. Lenasi, H.) (InTech, London, 2018). https://doi.org/10.5772/intechopen.74029

21. Pereira, L., Medina, R., Baena, M., Planas, A. M. \& Pozas, E. IFN gamma regulates proliferation and neuronal differentiation by STAT1 in adult SVZ niche. Front. Cell Neurosci. 9, 270 (2015).

22. Au-Yeung, N., Mandhana, R. \& Horvath, C. M. Transcriptional regulation by STAT1 and STAT2 in the interferon JAK-STAT pathway. JAK-STAT 2, e23931 (2013).

23. Refaeli, Y., Van Parijs, L., Alexander, S. I. \& Abbas, A. K. Interferon $\gamma$ is required for activation-induced death of T lymphocytes. J. Exp. Med. 196, 999-1005 (2002).

24. Sun, Y. et al. Regulation of XAF1 expression in human colon cancer cell by interferon beta: Activation by the transcription regulator STAT1. Cancer Lett. 260, 62-71 (2008).

25. Hoglund, P. J., Nordstrom, K. J. V., Schioth, H. B. \& Fredriksson, R. The solute carrier families have a remarkably long evolutionary history with the majority of the human families present before divergence of bilaterian species. Mol. Biol. Evol. 28, 1531-1541 (2011).

26. Bar-Peled, O. et al. Distribution of glutamate transporter subtypes during human brain development. J. Neurochem. 69, 2571-2580 (1997).

27. Rueda, C. B. et al. Mitochondrial ATP-Mg/Pi carrier SCaMC-3/Slc25a23 counteracts PARP-1-dependent fall in mitochondrial ATP caused by excitotoxic insults in neurons. J. Neurosci. 35, 3566-3581 (2015).

28. Chowanadisai, W., Graham, D. M., Keen, C. L., Rucker, R. B. \& Messerli, M. A. Neurulation and neurite extension require the zinc transporter ZIP12 (slc39a12). Proc. Natl. Acad. Sci. 110, 9903-9908 (2013).

29. Roberts, L. M. et al. Expression of the thyroid hormone transporters monocarboxylate transporter-8 ( SLC16A2) and organic ion transporter-14 ( SLCO1C1) at the blood-brain barrier. Endocrinology 149, 6251-6261 (2008).

30. Shin, B.-C. et al. Neural deletion of glucose transporter isoform 3 creates distinct postnatal and adult neurobehavioral phenotypes. J. Neurosci. 38, 9579-9599 (2018).

31. Saunders, N. R., Daneman, R., Dziegielewska, K. M. \& Liddelow, S. A. Transporters of the blood-brain and blood-CSF interfaces in development and in the adult. Mol. Aspects Med. 34, 742-752 (2013).

32. Tarling, E. J., de Aguiar Vallim, T. Q. \& Edwards, P. A. Role of ABC transporters in lipid transport and human disease. Trends Endocrinol. Metab. 24, 342-350 (2013).

33. Goasdoué, K., Miller, S. M., Colditz, P. B. \& Björkman, S. T. Review: The blood-brain barrier; protecting the developing fetal brain. Placenta 54, 111-116 (2017).

34. Niaudet, C. et al. Gpr116 receptor regulates distinctive functions in pneumocytes and vascular endothelium. PLoS ONE 10, e0137949 (2015).

35. Niwa, H., Miyazaki, J. \& Smith, A. G. Quantitative expression of Oct-3/4 defines differentiation, dedifferentiation or self-renewal of ES cells. Nat. Genet. 24, 372-376 (2000). 
36. Lin, H.-H. et al. Neuronatin promotes neural lineage in ESCs via Ca ${ }^{2+}$ signaling. Stem Cells 28, 1950-1960 (2010).

37. Carpenter, S. et al. TRIL, a functional component of the TLR4 signaling complex, highly expressed in brain. J. Immunol. 183, 3989-3995 (2009).

38. Harada, J., Foley, M., Moskowitz, M. A. \& Waeber, C. Sphingosine-1-phosphate induces proliferation and morphological changes of neural progenitor cells. J. Neurochem. 88, 1026-1039 (2004).

39. Shen, F., Song, C., Liu, Y., Zhang, J. \& Wei Song, S. IGFBP2 promotes neural stem cell maintenance and proliferation differentially associated with glioblastoma subtypes. Brain Res. 1704, 174-186 (2019).

40. Galli, A. Glypican 4 modulates FGF signalling and regulates dorsoventral forebrain patterning in Xenopus embryos. Development 130, 4919-4929 (2003).

41. Vernon, C. G. \& Swanson, G. T. Neto2 assembles with kainate receptors in DRG neurons during development and modulates neurite outgrowth in adult sensory neurons. J. Neurosci. 37, 3352-3363 (2017).

42. Helms, H. C. C., Nielsen, C. U., Waagepetersen, H. S. \& Brodin, B. Glutamate transporters in the blood-brain barrier. In Glial Amino Acid Transporters (eds Ortega, A. \& Schousboe, A.) 297-314 (Springer International Publishing, Berlin, 2017). https://doi. org/10.1007/978-3-319-55769-4_15.

43. Betsholtz, C. Lipid transport and human brain development. Nat. Genet. 47, 699-701 (2015).

44. Nguyen, L. N. et al. Mfsd2a is a transporter for the essential omega-3 fatty acid docosahexaenoic acid. Nature 509, 503-506 (2014).

45. Alcudia, J. F. Lysyl oxidase and endothelial dysfunction: Mechanisms of lysyl oxidase down-regulation by pro-inflammatory cytokines. Front. Biosci 13, 2721 (2008).

46. Zheng, Z. et al. Reprogramming of human fibroblasts into multipotent cells with a single ECM proteoglycan, fibromodulin. Biomaterials 33, 5821-5831 (2012).

47. Whelan, M. C. \& Senger, D. R. Collagen I initiates endothelial cell morphogenesis by inducing actin polymerization through suppression of cyclic AMP and protein kinase A. J. Biol. Chem. 278, 327-334 (2003).

48. Dunwoodie, S. L. The role of hypoxia in development of the mammalian embryo. Dev. Cell 17, 755-773 (2009).

49. Ho, S.-Y. et al. SDF-1/CXCR4 signaling maintains stemness signature in mouse neural stem/progenitor cells. Stem Cells Int. 2017, $1-14$ (2017).

50. Rey, S. \& Semenza, G. L. Hypoxia-inducible factor-1-dependent mechanisms of vascularization and vascular remodelling. Cardiovasc. Res. 86, 236-242 (2010).

51. Bem, J. et al. Wnt/ $\beta$-catenin signaling in brain development and mental disorders: Keeping TCF7L2 in mind. FEBS Lett 593, 1654-1674 (2019).

52. Daneman, R. et al. Wnt/-catenin signaling is required for CNS, but not non-CNS, angiogenesis. Proc. Natl. Acad. Sci. 106, 641-646 (2009).

53. Sabbagh, M. F. et al. Transcriptional and epigenomic landscapes of CNS and non-CNS vascular endothelial cells. eLife 7, e36187 (2018).

54. Eggenberger, J., Blanco-Melo, D., Panis, M., Brennand, K. J. \& ten Oever, B. R. Type I interferon response impairs differentiation potential of pluripotent stem cells. PNAS 116, 1384-1393 (2019).

55. Lee, H.-C., Tan, K.-L., Cheah, P.-S. \& Ling, K.-H. Potential role of JAK-STAT signaling pathway in the neurogenic-to-gliogenic shift in down syndrome brain. Neural Plast. https://doi.org/10.1155/2016/7434191 (2016).

56. Thompson, C. L. et al. A high-resolution spatiotemporal atlas of gene expression of the developing mouse brain. Neuron $\mathbf{8 3}, 309-323$ (2014).

57. Varfolomeev, E. E. et al. Targeted disruption of the mouse caspase 8 gene ablates cell death induction by the TNF receptors, Fas/ Apo1, and DR3 and is lethal prenatally. Immunity 9, 267-276 (1998).

58. Xia, Y., Novak, R., Lewis, J., Duckett, C. S. \& Phillips, A. C. Xaf1 can cooperate with TNFa in the induction of apoptosis, independently of interaction with XIAP. Mol. Cell Biochem. 286, 67-76 (2006).

59. Xiao, S. et al. RIG-G as a key mediator of the antiproliferative activity of interferon-related pathways through enhancing p21 and p27 proteins. Proc. Natl. Acad. Sci. 103, 16448-16453 (2006).

60. Perez-Asensio, F. J., Perpiñá, U., Planas, A. M. \& Pozas, E. Interleukin-10 regulates progenitor differentiation and modulates neurogenesis in adult brain. J. Cell Sci. 126, 4208-4219 (2013).

61. Foshay, K. M. \& Gallicano, G. I. Regulation of Sox2 by STAT3 initiates commitment to the neural precursor cell fate. Stem Cells Dev. 17, 269-278 (2008).

62. Stephan, A. H., Barres, B. A. \& Stevens, B. The complement system: An unexpected role in synaptic pruning during development and disease. Annu. Rev. Neurosci. 35, 369-389 (2012).

63. Gama Sosa, M. A. et al. Interactions of primary neuroepithelial progenitor and brain endothelial cells: Distinct effect on neural progenitor maintenance and differentiation by soluble factors and direct contact. Cell Res. 17, 619-626 (2007).

64. Chen, C.-H. et al. Study of cathepsin B inhibition in VEGFR TKI treated human renal cell carcinoma xenografts. Oncogenesis 8, 15 (2019).

65. Tamayo, P. et al. Interpreting patterns of gene expression with self-organizing maps: Methods and application to hematopoietic differentiation. PNAS 96, 2907-2912 (1999).

66. Reich, M. et al. GenePattern 2.0. Nat. Genet. 38, 500-501 (2006).

67. Supek, F., Bošnjak, M., Škunca, N. \& Šmuc, T. REVIGO summarizes and visualizes long lists of gene ontology terms. PLoS ONE 6, e21800 (2011)

\section{Ackonowledgements}

This work was supported in part by philanthropic support from Roberta and Stephen R. Weiner to the Beth Israel Deaconess Department of Surgery and NIH R21EB024308 to CF.

\section{Author contributions}

A.T., C.F. and R.V conceptualized the project. R.V, C.A. and M.A.C. performed experiments. F.C.K., M.B. and C.A. performed data analysis and prepared the figures. F.C.K, C.F. and A.T wrote the manuscript. J.M reviewed the project and the manuscript. All authors commented on the manuscript.

\section{Competing interests}

The authors declare no competing interests.

\section{Additional information}

Supplementary information is available for this paper at https://doi.org/10.1038/s41598-020-77297-3.

Correspondence and requests for materials should be addressed to A.J.T. 
Reprints and permissions information is available at www.nature.com/reprints.

Publisher's note Springer Nature remains neutral with regard to jurisdictional claims in published maps and institutional affiliations.

(c) (i) Open Access This article is licensed under a Creative Commons Attribution 4.0 International License, which permits use, sharing, adaptation, distribution and reproduction in any medium or format, as long as you give appropriate credit to the original author(s) and the source, provide a link to the Creative Commons licence, and indicate if changes were made. The images or other third party material in this article are included in the article's Creative Commons licence, unless indicated otherwise in a credit line to the material. If material is not included in the article's Creative Commons licence and your intended use is not permitted by statutory regulation or exceeds the permitted use, you will need to obtain permission directly from the copyright holder. To view a copy of this licence, visit http://creativecommons.org/licenses/by/4.0/.

(C) The Author(s) 2020 